\section{Transportadores de Glicose na Sindrome Metabólica}

\section{RESUMO}

A regulação da homeostasia intra e extra-celular da glicose está diretamente relacionada ao controle preciso da expressão dos genes que codificam as diferentes isoformas de proteínas transportadoras de glicose, as quais se expressam de maneira tecido-específica, em conseqüência do padrão de ativação dos fatores transcricionais reguladores de cada gene, em cada tipo celular. A síndrome metabólica (SM) abrange uma grande variedade de alterações fisiopatológicas, todas de repercussões sistêmicas, acometendo os mais distintos territórios do organismo, nos quais alterações nos transportadores de glicose presentes são observadas em maior ou menor grau. A presente revisão abordará as alterações na expressão de transportadores de glicose claramente demonstradas na literatura, cujas repercussões nos fluxos territoriais de glicose auxiliam na compreensão de mecanismos fisiopatológicos da SM, assim como dos tratamentos propostos para esta entidade. (Arq Bras Endocrinol Metab 2006;50/2:177-189)

Descritores: Transportador de glicose; GLUT; GLUT4; Síndrome metabólica; Resistência à insulina

\begin{abstract}
Glucose Transporters in the Metabolic Syndrome.

Intra- and extra-cellular glucose homeostasis is directly related to gene expression control of different isoforms, which encode different glucose transporters, being tissue-specifically expressed, as a consequence of transcriptional factors activity of each gene, in each cellular type. The metabolic syndrome (MS) comprises a wide range of physiopathological abnormalities, all involved in systemic changes, which occur in different territories of the body, where glucose transporters changes are observed in diverse degrees. The present review will describe the glucose transporters expression changes clearly shown in literature, which reflexes in glucose flow, and can facilitate the MS physiopathological understanding, as well as possible treatments for the syndrome. (Arq Bras Endocrinol Metab 2006;50/2:177-189)
\end{abstract}

Keywords: Glucose transporter; GLUT; GLUT4; Metabolic syndrome; Insulin resistance

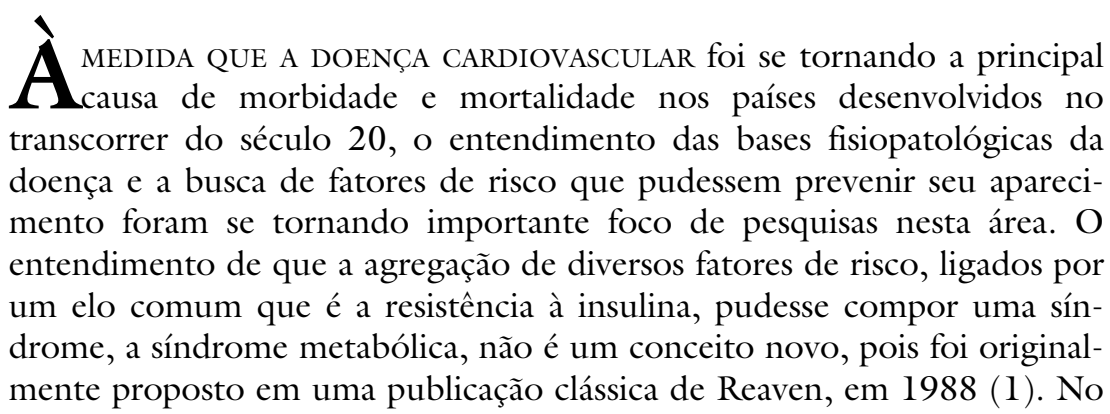

Ubiratan Fabres Machado Beatriz D. Schaan Patricia M. Seraphim

Departamento de Fisiologia e Biofísica, Instituto de Ciências Biomédicas da Universidade de São Paulo (UFM), São Paulo, SP; Serviço de Medicina Experimental da Unidade de Pesquisa do Instituto de Cardiologia /

Fundação Universitária de Cardiologia (BDS), Porto Alegre, RS; e Departmento de Biologia Marinha e Manejamento Costeiro, Universidade do Estado de São Paulo, UNESP São Vicente (PMS), São Vicente, SP.

Recebido em 10/12//05 Aceito em 17/01/06 
entanto, desde essa publicação até a atualidade, diversas modificações foram observadas em seus critérios diagnósticos, hipóteses quanto à sua etiologia, importância clínica de seu diagnóstico e mecanismos fisiopatológicos envolvidos.

Uma interação entre RI, inflamação e obesidade é a hipótese fisiopatológica que mais amplamente unifica os diversos itens da síndrome. A resistência à insulina correlaciona-se com o grau de obesidade, especialmente a obesidade abdominal, e é forte fator preditivo para o desenvolvimento de diabetes, assim como o é a presença de processo inflamatório difuso e a obesidade. A insulina, além de seus clássicos efeitos na homeostase glicêmica, também apresenta atividades anti-inflamatórias, de forma que um estado de resistência à insulina não apenas reduz a utilização de glicose pelos tecidos insulino-sensíveis, mas também estimula a sinalização pró-inflamatória (2).

A resistência à insulina tem como mais importante contribuinte o excesso de ácidos graxos livres (AGL) circulantes, os quais se originam do tecido adiposo e das lipoproteínas ricas em triglicerídeos. Em acordo com as ações anti-lipolítica e de estímulo à lípase lipoprotéica da insulina, a resistência a este hormônio é determinante de lipólise e aumento de AGL. No fígado, os AGL aumentam a produção de glicose, triglicerídeos e lipoproteínas de baixa densidade (VLDL), associandose redução do colesterol contido na lipoproteína de alta densidade (HDL-c) e aumento da densidade das lipoproteínas de baixa densidade (LDL). No músculo, os AGL reduzem a sensibilidade à insulina, inibindo a captação de glicose insulino-mediada. Dados epidemiológicos demonstrando papel preditivo dos AGL no desenvolvimento do diabetes, suportam os dados fisiopatológicos descritos (3). Aumento da glicose circulante e liberação de AGL aumentam a secreção de insulina do pâncreas, resultando em hiperinsulinemia, a qual pode levar à retenção de sódio e aumento da atividade simpática (2), contribuintes da hipertensão.

Tanto o tecido adiposo como outras células secretam excessivamente interleucina-6 (IL-6), fator de necrose tumoral alfa (TNF- $\alpha$ ) e outras citocinas, além de haver redução da adiponectina, citocina com propriedades anti-inflamatórias. Essas alterações resultam em maior resistência à insulina e lipólise, aumentando AGL e produção hepática de glicose, além da produção de fibrinogênio e plasminogen activator inhibitor-1 (PAI-1) pelo fígado, resultando em estado pró-trombótico (2).

A SM abrange uma grande variedade de alterações fisiopatológicas, todas de repercussões sistêmicas, acometendo os mais distintos territórios do orga- nismo, nos quais alterações nos transportadores de glicose presentes são observadas em maior ou menor grau. Embora não sejam muitos, na literatura, os estudos que associam diretamente SM e alterações de transportadores, à medida que estendemo-nos aos principais componentes da SM isoladamente, tais como obesidade, RI, DM e hipertensão, por exemplo, o espectro da literatura se expande. Por esses motivos, a presente revisão abordará apenas alterações de transportadores claramente demonstradas, cujas repercussões nos fluxos territoriais de glicose auxiliem na compreensão de mecanismos fisiopatológicos da SM.

\section{TRANSPORTADORES DE GLICOSE}

Os transportadores de glicose são uma família de 14 membros, os quais permitem a difusão facilitada de glicose, por gradiente de concentração, através da membrana plasmática das células. A figura 1 mostra a estrutura molecular básica dessas proteínas de membrana. Essas proteínas de 50-60 kDa, denominadas GLUTs 1 a 14 em ordem cronológica de caracterização $(4,5)$, são expressas de forma tecido e célula-específicos, apresentando propriedades cinéticas e reguladoras distintas que refletem seus papéis definidos no metabolismo celular da glicose e homeostase glicêmica corporal total. Além disso, a função de uma mesma isoforma pode ser diferente de um tecido para outro, em conseqüência do processo de diferenciação celular.

Embora 14 isoformas de transportadores já tenham sido caracterizadas, as primeiras 5 variantes descritas parecem ser as principais, e têm sido foco de estudos que buscam caracterizar os fluxos de glicose, tanto em situações fisiológicas como fisiopatológicas.

\section{TRANSPORTADORES DE GLICOSE E HOMEOSTASIA GLICÊMICA}

A ligação entre hiperglicemia e complicações microvasculares do diabetes é incontestável, especialmente após a publicação do estudo DCCT, há mais de uma década atrás (6). No entanto, o princípio de que a captação de glicose pelas células é diretamente proporcional aos seus níveis extra-celulares não é verdadeiro para muitas células e tecidos, que efetivamente apresentam redução da captação de glicose em resposta à hiperglicemia. De fato, a falha do tecido muscular e outros tecidos insulino-sensíveis em aumentar a captação de glicose em face de seus níveis plasmáticos elevados é fator contribuinte para a hiperglicemia do diabetes. 


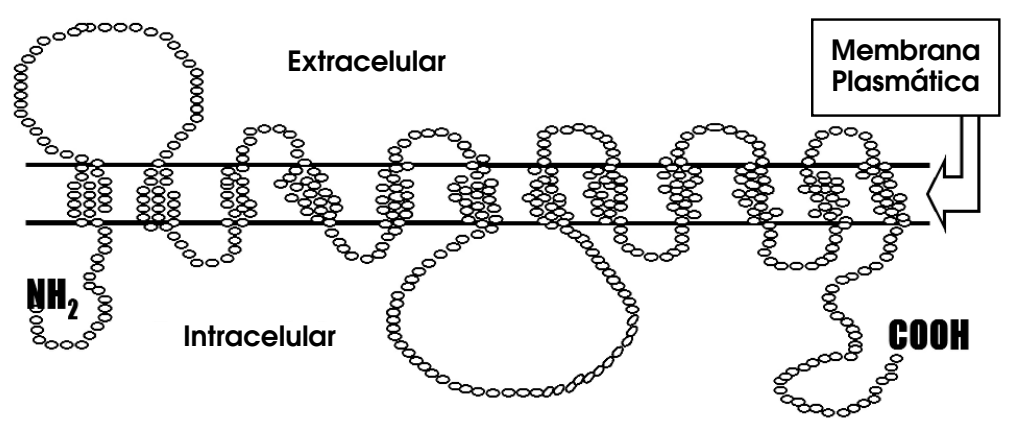

Figura 1. Estrutura bidimensional das proteínas transportadoras de glicose por difusão facilitada (GLUTS), determinada por análise hidropática dos segmentos de aminoácidos. Todas as isoformas possuem 12 segmentos transmembrânicos, hidrofóbicos, inseridos na porção lipídica da membrana plasmática, cujos aminoácidos formam alfahélices. Os segmentos transmembrânicos estão ligados por alças de conexão, e as terminacões NH2 e COOH localizam-se no intracelular. Nos GLUTs, as seqüências transmembrânicas são muito homólogas, enquanto as alças de conexão e as terminações são altamente heterólogas, determinando as especificidades de cada isoforma.

GLUT4 é o chamado transportador de glicose insulino-sensível, cujo principal papel é proporcionar a captação de glicose insulino-mediada em tecidos adiposo e muscular, tecidos que expressam especificamente, mas não unicamente, a proteína GLUT4. Modificações na expressão deste gen (ou gene), tanto em tecido adiposo quanto em músculo esquelético, correlacionam-se de maneira direta com aumento ou redução da sensibilidade insulínica (7).

Nas células em repouso, o GLUT4 localiza-se principalmente no compartimento intracelular, representando em adipócitos até $95 \%$ do conteúdo celular total deste transportador. O estímulo insulínico determina a movimentação de GLUT4 deste compartimento, e sua translocação em direção à membrana plasmática agudamente aumenta a captação de glicose, participando de forma importante no controle da homeostase glicêmica em nível tecidual e plasmático (8). Esse mecanismo torna a captação de glicose em músculo e tecido adiposo dependente da transmissão do sinal insulínico, e alterações nessa via, assim como a interferência de outros hormônios como a angiotensina, têm sido amplamente investigadas $(9)$.

O transportador GLUT4 tem sido investigado há mais de uma década em vários modelos experimentais, assim como em humanos com obesidade, RI, DM e hipertensão, todos componentes da SM. Adicionalmente, alteração na expressão do GLUT4 e de outros genes relacionados com a atividade metabólica do teci- do adiposo foi descrita recentemente no rato WOKW (Wistar Ottawa Karlburg W), que desenvolve SM com RI a partir de herança poligênica (10).

\section{GLUT4 em obesidade, resistência insulínica e DM2}

A expressão de GLUT4 em tecido muscular esquelético tem sido investigada em vários modelos de obesidade, apresentando resultados nem sempre concordantes. Nosso grupo descreveu redução do GLUT4 em músculo esquelético de camundongos obesos por tratamento com aurotioglicose (hiperfágicos) ou glutamato monossódico (normofágicos), e o mesmo foi observado em ratos Zucker diabéticos (11-13).

Em ratos, sabe-se que o aumento da idade é caracterizado pelo desenvolvimento espontâneo de um quadro de obesidade, que se acompanha de RI (12) de forma que o estudo dessa espécie é um modelo interessante para avaliar essas alterações idade-dependentes. Redução no conteúdo de proteína total de GLUT4 em tecido muscular foi inicialmente observada em ratos obesos de 20 meses de idade (14), o que foi posteriormente evidenciado ocorrer em ratas Long-Evans, já aos 10 meses de idade, permanecendo até 25 meses de idade (15). O mesmo já foi observado em músculo esquelético de humanos idosos, e relacionado à instalação de RI (16). Além disso, em humanos com DM2, a RI envolve também um defeito na translocação do GLUT4 para o sarcolema e 
túbulos T (17). Portanto, tanto redução no conteúdo de GLUT4 como defeito na sua translocação do intracelular para a membrana celular foram observados em modelos animais e em humanos com obesidade e/ou RI e/ou DM2.

No tecido adiposo, redução na expressão de GLUT4 associa-se com obesidade e desenvolvimento da RI, independentemente da regulação que ocorrer em músculo esquelético (18). Esta regulação negativa do GLUT4 em tecido adiposo é observada de forma consistente em estados de RI, tais como obesidade, DM2 e síndrome metabólica. Klip e cols., num estudo de revisão, relataram redução de mRNA e proteína GLUT4 no tecido adiposo branco de vários modelos animais de obesidade e diabetes (19). Nós observamos uma marcada redução de $70 \%$ nos conteúdos de mRNA e proteína GLUT4 em tecido adiposo branco de ratos Wistar idosos espontaneamente obesos (20). Além disso, demonstramos, em camundongos obesos com RI e DM2 $(11,21)$ e em ratos obesos com RI (22), que a redução no conteúdo de proteína GLUT4 do tecido adiposo ocorre tanto na membrana plasmática como no estoque intracelular.

Por outro lado, alguns estudos em tecido adiposo evidenciaram um incremento no conteúdo de GLUT4 em ratos obesos, ou nenhuma alteração, o que sugeria que o grau de obesidade e o padrão de sensibilidade à insulina pudessem ser fatores importantes a serem considerados quando avaliado o conteúdo de transportador de glicose. Neste sentido, um importante estudo de Papa e cols. (22) revelou que, durante o processo de desenvolvimento da obesidade, inicialmente, quando o ganho de peso é acelerado, há aumento na sensibilidade à insulina e no GLUT4 no tecido adiposo; posteriormente, quando a obesidade se estabiliza, sobrevém redução no GLUT4 no tecido adiposo e a RI se manifesta.

Embora o músculo esquelético seja o principal sítio de captação de glicose estimulada pela insulina, uma série de estudos vem apresentando provas convincentes de que a RI se iniciaria no tecido adiposo, e que este tecido teria um papel importante na sua indução em músculo esquelético e fígado (23). Os adipócitos produzem vários peptídeos bioativos, as adipocinas (TNF- $\alpha$, IL-6 e resistina, que reduzem a ação da insulina e adiponectina, que tem ação contrária), bem como AGL, que podem influenciar significativamente o transporte de glicose para as células, competindo como substrato energético utilizado especialmente nos músculos oxidativos aeróbios. Esta produção aumenta muito na obesidade e encontra-se associada à geração da RI nesta condição, uma vez que inibe a via de sinalização da insulina e a translocação do GLUT4 para a membrana plasmática (24-26), assim como pode reprimir a expressão de GLUT4 em miócitos e adipócitos (27).

Ainda, embora redução no conteúdo de GLUT4 em tecido adiposo sem alteração em músculo esquelético tenha sido observada em humanos com obesidade e/ou DM2, outras observações reforçam o papel chave do GLUT4 no tecido adiposo sobre a sensibilidade à insulina. Em animais transgênicos, a redução de GLUT4 seletivamente em tecido adiposo causa intolerância à sobrecarga glicídica e RI, embora haja expressão normal de GLUT4 em tecido muscular esquelético (28). Inversamente, em animais transgênicos com expressão elevada de GLUT4 seletivamente em tecido adiposo, ocorre um aumento global na sensibilidade à insulina e tolerância à glicose, mesmo na presença de diabetes $(29,30)$. Finalmente, estudo recente em camundongos knockout para GLUT4 em tecido muscular, e que apresentam hiperglicemia em resposta à sobrecarga glicídica, evidenciou que a superexpressão de GLUT4 no tecido adiposo branco normaliza a resposta glicêmica à sobrecarga de glicose, ressaltando a importância do GLUT4 no tecido adiposo para a manutenção do controle glicêmico (31). Assim, pode-se observar que o tecido adiposo, embora colabore com apenas $20 \%$ do total de captação de glicose no organismo, pode influenciar por via indireta (através de seus produtos) a sensibilidade à insulina de vários tecidos, e alterar de maneira importante a homeostasia glicídica.

Se por um lado, tanto em humanos como na maioria dos modelos experimentais, RI associa-se com obesidade, algumas situações de RI sem obesidade também já mostraram que se acompanham de redução na expressão do GLUT4, tais como gestação, DM e síndrome dos ovários policísticos. Nosso grupo, utilizando modelo animal de RI sem obesidade (ratos pinealectomizados), mostrou que a falta de melatonina induz redução do GLUT4 em tecido adiposo e muscular $(32,33)$. Essas observações reforçam a associação entre redução do GLUT4 e RI, independente da presença da obesidade.

Pelo descrito acima, observa-se que o espectro de alterações do GLUT4, que se reflete na homeostase glicêmica, é amplo, mas segue um padrão geral no qual a expressão do GLUT4 aumenta em estágios iniciais do desenvolvimento da obesidade, e diminui com a RI característica da obesidade estabelecida. A magnitude dessa redução é variável, e parece associar-se ao grau de RI, sendo mais acentuada nas situações em que o DM está clinicamente estabelecido. 


\section{GLUT4 na hipertensão}

Hipertensão está freqüentemente associada à RI, e alterações na expressão do GLUT4 já foram evidenciadas em vários modelos experimentais de hipertensão, tais como SHR - Spontaneously Hypertensive Rats (34) ou rato hipertenso Milan (35). Adicionalmente, redução na translocação da proteína GLUT4 induzida por insulina também foi observada em SHR (36). Por outro lado, há relato de aumento de proteína GLUT4 em membrana plasmática de músculo esquelético de SHR jovens (37), o que diminui com o avançar de idade, sugerindo um mecanismo semelhante ao observado no desenvolvimento da obesidade, no qual precocemente ocorre aumento na expressão do gene do GLUT4.

Inversamente ao padrão geral de regulação do GLUT4 na hipertensão, nós demonstramos na hipertensão induzida por ingestão excessiva de cloreto de sódio um aumento na expressão do GLUT4 em tecido adiposo e músculo esquelético, que se associou a aumento na sensibilidade à insulina, e foi atribuído à redução da angiotensina e/ou aumento da bradicinina (38). Nessa mesma linha, várias abordagens terapêuticas para hipertensão evidenciaram modular a expressão do GLUT4, o que se reflete em melhora da sensibilidade à insulina, e que será discutido adiante.

\section{GLUT4 e medidas terapêuticas}

Várias medidas terapêuticas que melhoram a sensibilidade à insulina envolvem aumento na expressão do gene do GLUT4, e conseqüentemente do conteúdo de proteína em tecido adiposo e/ou tecido muscular. Entre essas medidas encontram-se abordagens comportamentais, como emagrecimento por restrição calórica $(21,32)$ ou aumento na atividade física (39), e drogas sensibilizadoras da insulina (metformina e tiazolidinedionas, TZD) $(40,41)$.

A restrição de sal é recomendada como tratamento não farmacológico da hipertensão arterial, e seus efeitos sobre a sensibilidade à insulina têm sido estudados por nós e outros autores. Nosso grupo demonstrou que ratos submetidos à restrição crônica de sal, em comparação àqueles com sobrecarga de sal, apresentam menores níveis pressóricos e redução da captação de glicose insulino-mediada em adipócitos isolados de epidídimo (42). Em outro estudo (43), usando a técnica do clampe hiperinsulinêmico euglicêmico, mostramos que a restrição crônica de sal reduz, enquanto que a sobrecarga aumenta, a captação de glicose e a pressão arterial.

Ratos submetidos cronicamente à dieta pobre em sódio apresentam aumento de tecido adiposo epi- didimal (42), a qual representa gordura visceral, sabidamente relacionada à RI. De fato, o índice HOMA desses animais evidencia redução na sensibilidade à insulina (38). Desta forma, adipócitos do tecido adiposo visceral, particularmente resistentes aos efeitos anti-lipolíticos da insulina, liberam AGL na veia porta, expondo o fígado a elevadas concentrações desse substrato, o que já foi comentado ter um importante papel na gênese da RI. Nos animais submetidos à dieta pobre em sal não observamos alteração na expressão gênica de GLUT4, mas redução na translocação de GLUT4 insulino-mediada (38). Por outro lado, aumento na expressão do GLUT4 foi identificado naqueles animais submetidos a dietas com alto teor de sódio, tanto no tecido adiposo como no músculo esquelético, o que se acompanhou de aumento da sensibilidade insulínica, a despeito da hipertensão induzida pelo sal (38).

O uso do captopril, um inibidor da enzima conversora da angiotensina, em animais submetidos à restrição salina foi capaz de melhorar sua sensibilidade à insulina, enquanto que a inibição dos receptores ATl com losartan não apresentou o mesmo efeito $(42,43)$, sugerindo que o efeito da droga poderia ser conseqüência de aumento de bradicinina mais do que propriamente da inibição dos efeitos da angiotensina II. Todos esse mecanismos podem envolver passos-chave da sinalização insulínica (38).

Muitas investigações têm se focalizado na possibilidade de haver defeito no mecanismo de translocação do GLUT4 induzido pela insulina nos estados de resistência, especialmente quando a transdução do sinal insulínico está reduzida. Entretanto, nenhum estudo até hoje evidenciou que estados de RI podem se perpetuar apenas às expensas de translocação reduzida de GLUT4, sem conduzirem ao longo do tempo a uma redução na proteína GLUT4. Em contrapartida, já foi demonstrado em camundongos que superexpressam GLUT4, que o aumento deste transportador, per se, induz maior quantidade de proteína presente na membrana plasmática (44), confirmando que a quantidade de proteína transportadora funcionante, isto é, presente na membrana celular, depende fundamentalmente do conteúdo celular total, determinado pelo padrão de expressão do gene.

Todas as observações acima reforçam a importância da expressão do gene do GLUT4 na regulação da sensibilidade à insulina nos tecidos muscular e adiposo, e conseqüentemente na regulação da homeostasia glicêmica. De fato, camundongos transgênicos que superexpressam GLUT4 apresentam glicemias mais baixas e elevada tolerância à sobrecarga glicídica (44). Assim, a 
investigação dos mecanismos regulatórios da expressão do gene do GLUT4 deverá proporcionar novas abordagens preventivas ou terapêuticas para a RI. Neste sentido, demonstramos recentemente que o gene do GLUT4 é regulado por vários fatores transcricionais, que são acionados de maneira distinta, conforme o mecanismo indutor de regulação desse gene. Em músculo esqueléti$\mathrm{co}$, in vitro, ocorre rápido aumento do mRNA do GLUT4 em resposta à insulina, por inibição do NFK-B, um repressor do gene do GLUT4. O aumento do mRNA do GLUT4 também ocorre em resposta à atividade contrátil, por ativação dos MEF2A, MEF2D, e HIF-la, um estimulador do gene do GLUT4 (45).

\section{TRANSPORTADORES DE GLICOSE E ALTERAÇŌES TERRITORIAIS ESPECÍFICAS}

\section{GLUT2 no fígado}

Os hepatócitos expressam predominantemente a isoforma GLUT2, um transportador de alta capacidade de transporte de glicose (alto $\mathrm{Km}$ ), responsável no fígado pelo influxo do substrato no período pós-prandial, e pelo efluxo da glicose no período pós-absortivo e no jejum (46).

Em situações de hiperglicemia, o GLUT2 aumenta no fígado, o que decorre da ativação do fator transcricional SREBP - Sterol Response Element-Binding Protein (47). Por outro lado, recentes análises correlacionam a SM com a esteatose hepática não alcoólica (NASH, Non-Alcoholic Steatohepatitis). Esses estudos não apenas correlacionam estas duas entidades (48) como propõem a SM como um marcador da $\mathrm{NASH}$ (49). A NASH incide principalmente em pacientes portadores de alterações típicas da SM, especialmente a RI, e alguns mecanismos envolvidos na resistência hepática à insulina da NASH já foram caracterizados (50). Nesta patologia, espera-se que o aumento na expressão do GLUT2 hepático ocorra, conforme claramente demonstrado na esteatose hepática induzida em células humanas HepG2 incubadas, in vitro, em alta concentração de ácido oléico (51).

Entretanto, repercussão funcional do aumento do GLUT2 hepático em DM não foi demonstrada, provavelmente porque esse transportador não impõe restrição alguma ao transporte de glicose (alto $\mathrm{Km}$ ), e, portanto, o aumento da sua expressão não refletiria em aumento do transporte. No mesmo sentido, já demonstramos que é possível aumentar a sensibilidade à insulina no fígado, aumentando a síntese de glicogênio hepático estimulada pelo hormônio, sem alterar a expressão do GLUT2 (52).
Assim, aumento do GLUT2 hepático em estados que fazem parte da SM parece decorrer de mecanismos regulatórios da expressão do gene acionados pela RI, mas o significado fisiopatológico do aumento do GLUT2 em hepatócito, se existente, ainda não foi demonstrado.

\section{GLUT2 na célula B pancreática}

A secreção de insulina pela célula $B$ pancreática em resposta à glicose depende do metabolismo intracelular desse substrato. A célula B pancreática expressa a isoforma GLUT2 de transportador de glicose, cuja alta capacidade de transporte permite que as elevações de glicose plasmática se reflitam em variações paralelas na concentração intracelular da glicose, assim garantindo uma resposta secretória proporcional às variações plasmáticas (7).

Sabe-se que estados de RI, por estimularem hipersecreção de insulina, induzem, em longo prazo, redução na capacidade secretória da célula $B(53)$. A perda de capacidade secretória da insulina ocorre principalmente em resposta à glicose, e redução na expressão do GLUT2 em ilhotas pancreáticas de diabéticos já foi claramente demonstrada (54).

Prejuízo na secreção de insulina tem sido também atribuído a um efeito lipotóxico, causado especialmente pelo ácidos graxos saturados de cadeia longa. Isso pôde ser observado em ilhotas pancreáticas isoladas de ratos tratados cronicamente com dieta rica em ácidos graxos saturados de cadeia longa (gordura animal), onde ocorre uma redução de $50 \%$ na secreção de insulina induzida por glicose, assim como no conteúdo da proteína GLUT2 (55). Por outro lado, já foi demonstrado que as TZD, que melhoram a sensibilidade à insulina e a dislipidemia, induzem aumento da expressão do GLUT2 na célula B pancreática (56).

Assim, parece evidente que mecanismos fisiopatológicos da SM possam induzir redução na expressão do GLUT2 em células B pancreáticas, comprometendo a secreção de insulina em resposta à glicose, contribuindo para o estabelecimento de estados de intolerância à glicose.

\section{GLUT4 no coração}

No coração com oxigenação normal, os AGL são a principal fonte de energia, seguidos da glicose, lactato, cetonas e aminoácidos, que representam rápida adaptação dos cardiomiócitos de acordo com o substrato presente em maior abundância. A glicose é o principal substrato utilizado na isquemia, sobrecarga de trabalho e de pressão. Assim como no músculo esquelético, seu transporte é realizado principalmente 
pelo GLUT4, cuja translocação do citosol à membrana plasmática é especialmente estimulada pela contração continuada dos cardiomiócitos, representando a insulina importante estímulo adicional nesse processo (57). Aparentemente, o GLUTl tem papel na manutenção da captação basal de glicose pelos cardiomiócitos, podendo ter papel mais importante, no entanto, na ausência de GLUT4 (58), na isquemia miocárdica (59) e no jejum prolongado (60).

Os estímulos contrátil e hipóxico levam à translocação de GLUT4 à membrana plasmática pela via sinalizadoras da AMPK (proteína quinase AMP-ativada) $(61,62)$. Já a insulina aumenta a captação de glicose por maior translocação de GLUT4 via fosfatidilinositol 3-quinase (PI3k) e fosforilação/ativação de Akt (63).

No tratamento crônico de camundongos com glutamato monossódico, observamos, associada à RI, redução na expressão de GLUT4 em tecido adiposo (70\%), músculo esquelético (40\%) e cardíaco (28\%) após estabelecida a obesidade (7 meses) (22). Condições de RI ou DM caracterizam-se por um excesso de metabolismo de AGL também no coração, o que se traduz em piora da função contrátil. Esta pode ser restaurada pelo aumento da expressão de GLUT4, como foi demonstrado em coração isolado de camundongos transgênicos com DM associado à maior expressão de GLUT4 (64), caracterizando a importância do adequado metabolismo da glicose no cardiomiócito. O próprio excesso de AGL interfere no transporte de glicose, já que uma maior disponibilidade de AGL induzida por sua infusão intravenosa reduz a expressão de GLUT4 no músculo cardíaco com conseqüente depressão do transporte de glicose insulino-mediado, sem alterar a expressão de FAT/CD36 (transportador de AGL) (65). Em ratos com $\mathrm{RI}$ induzida por dieta rica em frutose, observa-se adaptação inadequada do cardiomiócito à isquemia, pois o fluxo glicolítico fica reduzido por menor translocação de GLUT4 à membrana celular do que se espera nessa condição (66).

Apesar de maior sensibilidade à insulina $\mathrm{e}$ expressão de GLUT4 em tecidos muscular esquelético e adiposo serem observadas em ratos submetidos à dieta hipersódica, essas alterações não são observadas no coração, sugerindo modulação tecido-específica do GLUT4 neste modelo (38).

Interessante observar que indivíduos com SM apresentam anormalidades da geometria cardíaca (aumento das dimensões e massa do ventrículo esquerdo) (67), o que se associa à RI (68), alterações miocárdicas semelhantes às observadas em camundongos knockout para GLUT4 (69).
O uso de agentes farmacológicos da classe das TZDs em modelos animais de obesidade (ratos resistentes à insulina) mostrou normalização da redução da proteína GLUT4 e da captação de glicose pelo miocárdio (70). Aumento da translocação de GLUT4 do citoplasma à membrana plasmática foi observado por outros autores com a troglitazona (71). A promoção da captação e metabolismo da glicose, associada à redução de AGL circulantes e de sua captação pelos cardiomiócitos induzidos pelos mesmos agentes, seriam potencialmente benéficos em aumentar a tolerância à isquemia.

\section{GLUT4 na célula muscular lisa vascular (CMLV)}

Tem sido proposto que o GLUT4 seja o principal transportador de glicose na CMLV, onde desempenha papel importante na contração induzida por agonistas. Em aorta de camundongo isolada e desnudada de endotélio, $50 \%$ do transporte basal de glicose (não estimulado por insulina) é dependente de GLUT4. Embora a participação do GLUT4 na resposta contrátil aguda seja variável de acordo com o tipo de agonista, cronicamente parece que o transportador exerce um papel importante que se reflete na resposta contrátil da CMLV. Em aorta de camundongo hipertenso por excesso de angiotensina II (angiotensin II hypertensive mice) foi observada uma redução de $46 \%$ na expressão do GLUT4, e o knockout crônico do GLUT4 na CMLV está associado com aumento na reatividade vascular. Essas observações evidenciam que alterações na expressão do GLUT4 em CMLV determinam alterações funcionais que podem se associar com a hipertensão (72).

Redução na expressão do GLUT4 também já foi observada em CMLV de aorta e artéria carótida de ratos hipertensos DOCA-sal, induzindo redução da captação e metabolismo da glicose, o que deve participar das alterações contráteis dessas células em algumas formas de hipertensão (73). Além disso, o tratamento desses animais com TZDs (troglitazona e rosiglitazona) mostrou-se capaz de reduzir a pressão arterial e induzir aumento na expressão do GLUT4 em aorta. Adicionalmente, a troglitazona mostrou efeito em induzir em 24 horas a expressão do GLUT4 em cultura de aortas explantadas (74).

A possibilidade de a insulina modular agudamente o transporte de glicose via GLUT4 em CLMV também já foi confirmada. Em CLMV humanas em cultura foi demonstrado que a insulina estimula o transporte de glicose e a correspondente translocação do GLUT4 para membrana plasmática, mecanismo esse mediado por um aumento na síntese de óxido nítrico, o que estimula produção de GMP cíclico e ativação de proteínas quinases dependentes de GMP (75). 


\section{Célula endotelial}

Alterações funcionais das células endoteliais desempenham um papel-chave na patogênese das doenças vasculares decorrentes da SM e do DM. Estas alterações têm sido investigadas nos mais distintos territórios do organismo, nos quais vários mecanismos fisiopatológicos foram implicados na gênese das alterações, entre os quais podemos destacar: 1) ativação da via da PKC; 2) aumento de metaloproteinases teciduais; 3 ) alterações na rota metabólica da hexosamina; 4) redução na produção de óxido nítrico. Todos esses mecanismos etiopatogênicos devem ser acionados em resposta a alterações no metabolismo intracelular de glicose, uma vez que a hiperglicemia parece ser um evento-chave no desenvolvimento dessas alterações.

Dessa maneira, o aporte de glicose à célula endotelial também deve se alterar, o que poderia envolver alterações de transportadores de glicose. Entretanto, poucos estudos nesse sentido já foram realizados. GLUTl é o transportador de glicose mais característico da célula endotelial (7), e alterações importantes no transportador só foram identificadas em alguns territórios especiais.

Está bem caracterizado que ocorre uma redução do GLUTl em células endoteliais da barreira hematoencefálica em resposta à elevação glicêmica crônica (76). Este ajuste impede um fluxo transendotelial exacerbado de glicose através da barreira, protegendo o SNC de efeitos glicotóxicos. Por outro lado, a redução da proteína GLUTl na barreira hemato-encefálica é responsável pelo aparecimento de sinais centrais de hipoglicemia em diabéticos adaptados ao estado hiperglicêmico, e que sofrem quedas glicêmicas bruscas, mesmo que sejam preservados níveis periféricos de glicemia considerados satisfatórios.

Em células endoteliais da barreira hematoretiniana interna, nenhuma alteração da expressão do GLUT1 foi observada no DM (77), embora se saiba que o desenvolvimento da retinopatia esteja associado a aumento na concentração de glicose no interstício retiniano. Entretanto, defeito no estabelecimento das "tigh-junctions" entre as células endoteliais da retina, que garantem a característica funcional de barreira, já foi demonstrado em rato diabético, e correlacionado com redução na proteína ocludina, que participa da junção intercelular (78). Dessa maneira, compreende-se que a concentração intersticial de glicose aumente, em resposta à elevação glicêmica, a partir de fluxo intercelular, decorrente da perda da integridade funcional da barreira, independentemente de alteração no transporte de glicose trans-endotelial.
Finalmente, um recente estudo em endotélio de artéria coronária de ratos revelou a presença de várias isoformas de transportadores de glicose: do GLUT1 ao GLUT5 e ainda do co-transportador de sódio e glicose SGLTl (79). Verificou-se que a hiperglicemia reduz GLUT1, GLUT4 e GLUT5, aumenta GLUT2, sem alterar SGLT1. Considerando-se a alta capacidade de transporte do GLUT2, foi proposto que o seu aumento seria suficiente para tornar o endotélio dos vasos cardíacos suscetível à glicotoxicidade (79).

\section{GLUTs e SGLTs no rim}

A reabsorção tubular de glicose é um processo que ocorre nas células epiteliais do túbulo contorcido proximal, envolvendo 2 classes de transportadores de glicose: os co-transportadores de $\mathrm{Na}^{+}$-glicose (SGLTs) e os GLUTs. A glicose é transportada através do bordo em escova (luminal) da membrana celular pelos SGLTs, o que requer um gradiente eletroquímico, acumulando-se no citoplasma da células epiteliais (80); seu efluxo para o interstício ocorre através de sua membrana basolateral e é mediado pelos GLUTs, de acordo com gradiente de concentração. No segmento S1, SGLT2 e GLUT2, transportadores de baixa afinidade e alta capacidade estão expressos, de forma que a maior parte da glicose filtrada é reabsorvida nessa região $(80)$.

$\mathrm{O}$ aumento de glicose sangüínea e intersticial, característicos do DM, reduzem o gradiente de glicose túbulo-sangue, diminuindo seu efluxo das células epiteliais e, conseqüentemente, todo o processo de reabsorção. Nessa situação, portanto, faz-se necessário o aumento da expressão gênica desses transportadores, o que foi relatado na literatura em modelos animais de DM por nós $(81,82)$ e outros autores $(83,84)$. O tratamento com insulina cronicamente diminui a expressão de GLUT2, o que se deveria à redução da glicemia; no entanto, agudamente observa-se aumento da expressão desse transportador (85), o que poderia se dever a um efeito próprio da insulina. Além da glicose e insulina, o sistema nervoso simpático também está envolvido na modulação dos transportadores de glicose no rim, já que a desnervação renal cirúrgica causa redução na expressão de GLUT2, glicosúria e natriurese (86), enquanto que a hiperatividade simpática, característica dos animais SHR, causa exatamente alterações contrárias (82).

Diferentemente dos tecidos insulino-sensíveis, as células renais aumentam a captação de glicose à medida que sua concentração plasmática se eleva, como ocorre no DM. Nas células mesangiais renais, onde desenvolvem-se os eventos-chave relacionados à 
gênese da nefropatia diabética, identifica-se principalmente o GLUT1, tanto em camundongos (87) como em humanos (88). O aumento de sua expressão em córtex renal no diabetes foi por nós demonstrado in vivo (89), o que, em associação às alterações de GLUT2 acima descritas, aumentaria a captação de glicose pelas células mesangiais, acentuando as alterações causadas pelo DM. A associação de hipertensão arterial, que aproxima o modelo experimental da nefropatia diabética em humanos, aumenta ainda mais a expressão, tanto de GLUT1 como de GLUT2 (90), o que é compatível com a pior evolução clínica da nefropatia observada quando da hipertensão não controlada.

Hipertensão arterial, hiperinsulinemia e aumento de gordura visceral são situações de hiperatividade simpática, assim como o é a própria SM (91). Em considerando-se o aumento de expressão de GLUTs 1 e 2 em córtex renal induzidos pelo DM, hipertensão e hiperatividade simpática observados em nossos estudos, é possível que essas alterações estejam presentes em rins de indivíduos com SM, levando a um ciclo vicioso de maior elevação glicêmica, retenção de sódio e alterações homeostáticas decorrentes.

\section{CONSIDERAÇÕES FINAIS}

A regulação da homeostasia intra e extra-celular da glicose está diretamente relacionada ao controle preciso da expressão dos genes que codificam as diferentes isoformas de proteínas transportadoras de glicose, as quais se expressam de maneira tecido-específica, em conseqüência do padrão de ativação dos fatores transcricionais reguladores de cada gene, em cada tipo celular. Na Síndrome Metabólica, dependendo dos componentes presentes e da magnitude dos acometimentos, importantes alterações na expressão dos transportadores de glicose podem ser observadas, as quais determinam mudanças nos fluxos territoriais de glicose que concorrem para alterar a homeostasia extracelular da glicose e/ou o metabolismo intracelular do substrato, este último conduzindo a alterações funcionais locais (figura 2). Alterações do GLUT4 em tecido adiposo e muscular esquelético são fundamentais na sensibilidade tecidual à insulina, e repercutem em perda do controle glicêmico. Além disso, alterações na expressão de transportadores de glicose em outros tipos celulares podem determinar disfunções locais e/ou sistêmicas. Neste sentido, destacam-se: redução do GLUT2 em célula B pancreática, o que altera a secreção de insulina induzida por glicose; redução do
Transportadores de Glicose e Síndrome Metabólica
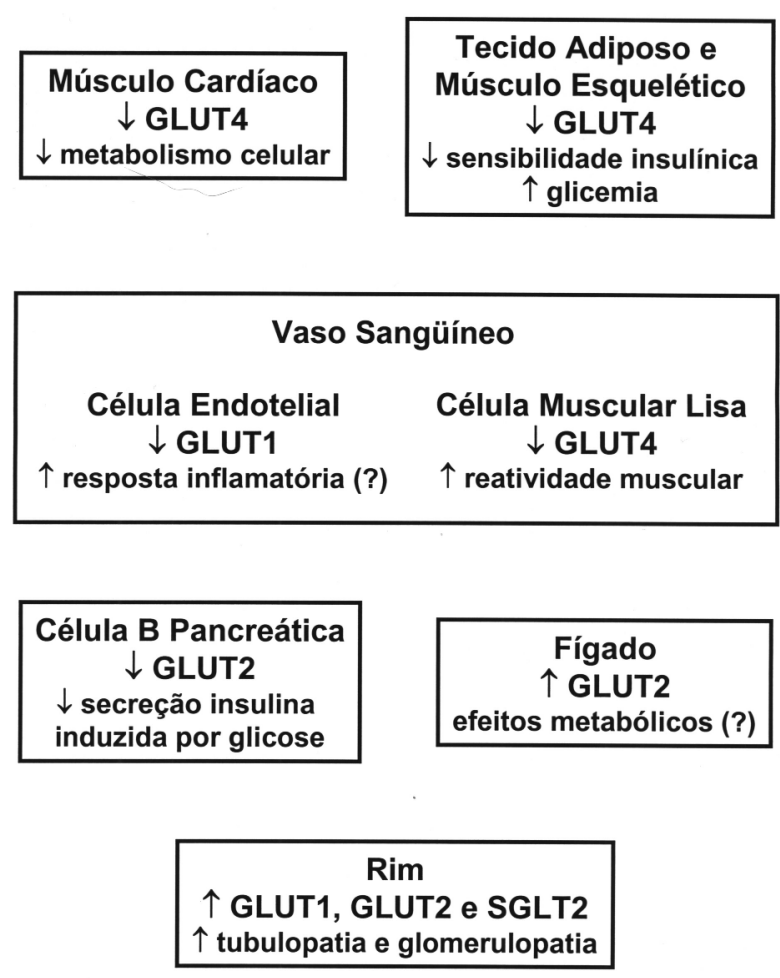

Figura 2. Principais alterações na expressão dos transportadores de glicose observadas na Síndrome Metabólica, respectivos territórios em que ocorrem, e repercussões fisiopatológicas que determinam. Os dados não são apresentados na forma de fluxograma, pois essas alterações não apresentam relação clara de causa-efeito, exceto pela participação da hiperglicemia (que envolve redução do GLUT4) como fator etiopatogênico. A existência de mecanismos moleculares comuns como indutores das alterações não deve ser descartada, os quais, modulados de forma independente em cada tipo celular, explicariam o fato de as alterações serem tecido-específicas.

GLUT4 em célula muscular lisa vascular, o que aumenta a contratilidade celular e o tono vascular; diminuição de GLUTl na célula endotelial da barreira hemato-encefálica, o que altera aporte de glicose ao interstício cerebral; diminuição de GLUT4 em célula muscular cardíaca, o que altera a capacidade oxidativa da fibra; e aumento de GLUT2 e SGLT2 em célula epitelial do túbulo renal e de GLUT1 em célula mesangial glomerular, o que contribui para o desenvolvimento de nefropatia. As condições metabólicohormonais em cada tipo celular podem determinar uma regulação diferencial na expressão de um mesmo gene, por exemplo, enquanto a expressão do GLUT2 diminui na célula $\mathrm{B}$ pancreática, aumenta em fígado e rim, com conseqüências específicas para cada território. Todas essas observações nos demonstram que 
importantes alterações nos fluxos de glicose em diferentes tipos celulares ocorrem na SM, participando do amplo espectro de alterações fisiopatológicas que a síndrome apresenta.

\section{REFERÊNCIAS}

1. Reaven GM. Banting lecture 1988. Role of insulin resistance in human disease. Diabetes 1988:37:1595-607.

2. Dandona P, Aljada A, Chaudhuri A, Mohanty P, Garg R. Metabolic syndrome: A comprehensive perspective based on interactions between obesity, diabetes, and inflammation. Circulation 2005; 111:1448-54.

3. Pankow JS, Duncan BB, Schmidt MI, Ballantyne CM, Couper DJ, Hoogeveen RC, et al. Fasting plasma free fatty acids and risk of type 2 diabetes: The atherosclerosis risk in communities study. Diabetes Care 2004:27:7782.

4. Scheepers A, Joost HG, Schurmann A. The glucose transporter families SGLT and GLUT: Molecular basis of norma and aberrant function. J Parenter Enteral Nutr 2004;28(5):364-71.

5. WU X, Freeze HH. GLUT14, a duplicon of GLUT3, is specifically expressed in testis as alternative splice forms. Genomics 2002;80:553-7.

6. The Diabetes Control and Complications Trial Research Group. The effect of intensive treatment of diabetes on the development and progression of long-term complications in insulin-dependent diabetes mellitus. N Engl J Med 1993:329:977-86.

7. Thorens B, Charron MJ, Lodish HF. Molecular physiology of glucose transporters. Diabetes Care 1990; 13:209-18.

8. Rea S, James DE. Moving GLUT4: The biogenesis and trafficking of GLUT4 storage vesicles. Diabetes 1997:46:1667-77.

9. Folli F, Saad MJ, Velloso L, Hansen $H$, Carandente $O$, Feener EP, et al. Cross-talk between insulin and angiotensin II signalling systems. Exp Clin Endocrinol Diabetes 1999; 107(2):133-9.

10. Kloting $\mathrm{N}$, Bluher $\mathrm{M}$, Kloting I. The polygenetically inherited metabolic syndrome of WOKW rats is associated with insulin resistance and altered gene expression in adipose tissue. Diabetes Metab Res Rev 2005; (Epub ahead of print).

11. Machado UF, Shimizu Y, Saito M. Decreased glucose transporter (GLUT 4) content in insulin-sensitive tissues of obese aurothioglucose- and monosodium glutamatetreated mice. Horm Metab Res 1993:25:462-5.

12. Machado UF, Shimizu I, Saito M. Reduced content and preserved translocation of glucose transporter (GLUT 4) in white adipose tissue of obese mice. Physiol Behav 1994;55:621-5

13. Handberg A, Kayser L, Hoyer PE, Voldstedlund $M$, Hansen HP. Vinten J. Metformin ameliorates diabetes but does not normalize the decreased GLUT 4 content in skeletal muscle of obese ( $\mathrm{fa} / \mathrm{fa}$ ) Zucker rats. Diabetologia 1993;36:481-6.
14. Lin JL, Asano T, Shibasaki Y, Tsukuda K, Katagiri H, Ishihara $\mathrm{H}$, et al. Altered expression of glucose transporter isoforms with aging in rats - selective decrease in GluT4 in the fat tissue and skeletal muscle. Diabetologia 1991:34:477-82.

15. Gulve EA, Henriksen EJ, Rodnick KJ, Youn JH, Holloszy JO. Glucose transporters and glucose transport in skeletal muscles of 1- to 25-mo-old rats. Am J Physiol 1993;264(3P+1):E319-27

16. Houmard JA, Weidner MD, Dolan PL, Leggett-Frazier N, Gavigan KE, Hickey MS, et al. Skeletal muscle GLUT4 protein concentration and aging in humans. Diabetes 1995;44:555-60.

17. Garvey WT, Maianu L, Zhu JH, Brechtel-Hook G, Wallace $P$, Baron $A D$. Evidence for defects in the trafficking and translocation of GLUT4 glucose transporters in skeletal muscle as a cause of human insulin resistance. J Clin Invest 1998:101:2377-86.

18. Shepherd PR, Kahn BB. Glucose transporters and insulin action - implications for insulin resistance and diabetes mellitus. N Engl J Med 1999;341:248-57.

19. Klip A, Tsakiridis T, Marette A, Ortiz PA. Regulation of expression of glucose transporters by glucose: A review of studies in vivo and in cell cultures. Faseb J 1994:8:4353.

20. Seraphim PM, Assis TGR, Volpato CB, Nunes MT, Machado UF. GLUT4 gene expression insulin-resistant obese rats involves post-transcriptional poly-A tails mRNA modulation. Diab Res Clin Prac 2000;50:S157-8.

21. Papa PC, Seraphim PM, Machado UF. Loss of weight restores GLUT 4 content in insulin-sensitive tissues of monosodium glutamate-treated obese mice. Int J Obes Relat Metab Disord 1997:21:1065-70.

22. de Carvalho Papa P, Vargas AM, da Silva JL, Nunes MT, Machado UF. GLUT4 protein is differently modulated during development of obesity in monosodium glutamate-treated mice. Life Sci 2002;71:1917-28.

23. Smith U. Impaired ('diabetic') insulin signaling and action occur in fat cells long before glucose intolerance - is insulin resistance initiated in the adipose tissue? Int J Obes Relat Metab Disord 2002;26:897-904.

24. Guerre-Millo M. Adipose tissue and adipokines: For better or worse. Diabetes Metab 2004;30:13-9.

25. Mora S, Pessin JE. An adipocentric view of signaling and intracellular trafficking. Diabetes Metab Res Rev 2002; 18:345-56.

26. Hotamisligil GS, Budavari A, Murray D, Spiegelman BM. Reduced tyrosine kinase activity of the insulin receptor in obesity-diabetes. Central role of tumor necrosis factor-alpha. J Clin Invest 1994;94:1543-9.

27. Hotamisligil GS, Spiegelman BM. Tumor necrosis factor alpha: a key component of the obesity-diabetes link. Diabetes 1994;43:1271-8.

28. Abel ED, Peroni O, Kim JK, Kim YB, Boss O, Hadro E, et al. Adipose-selective targeting of the GLUT4 gene impairs insulin action in muscle and liver. Nature 2001:409:729-33.

29. Tozzo E, Gnudi L, Kahn BB. Amelioration of insulin resistance in streptozotocin diabetic mice by transgenic overexpression of GLUT4 driven by an adipose-specific promoter. Endocrinology 1997;138:1604-11. 
30. Shepherd PR, Gnudi L, Tozzo E, Yang H, Leach F, Kahn BB. Adipose cell hyperplasia and enhanced glucose disposal in transgenic mice overexpressing GLUT4 selectively in adipose tissue. J Biol Chem 1993;268:22243-6.

31. Carvalho E, Kotani K, Peroni OD, Kahn BB. Adipose-specific overexpression of GLUT4 reverses insulin resistance and diabetes in mice lacking GLUT4 selectively in muscle. Am J Physiol Endocrinol Metab 2005;289:E551-61.

32. Zanquetta MM, Seraphim PM, Sumida DH, Cipolla-Neto J, Machado UF. Calorie restriction reduces pinealectomy-induced insulin resistance by improving GLUT4 gene expression and its translocation to the plasma membrane. J Pineal Res 2003;35:141-8.

33. Lima FB, Machado UF, Bartol I, Seraphim PM, Sumida DH, Moraes SM, et al. Pinealectomy causes glucose intolerance and decreases adipose cell responsiveness to insulin in rats. Am J Physiol 1998;275(6Pt1):E934-41.

34. Paternostro G, Clarke K, Heath J, Seymour AM, Radda GK. Decreased GLUT-4 mRNA content and insulin-sensitive deoxyglucose uptake show insulin resistance in the hypertensive rat heart. Cardiovasc Res 1995;30:205-11.

35. Campbell IW, Dominiczak AF, Livingstone C, Gould GW. Analysis of the glucose transporter compliment of metabolically important tissues from the Milan hypertensive rat. Biochem Biophys Res Commun 1995;21 1:780-91.

36. Chiappe De Cingolani GE, Caldiz Cl. Insulin resistance and GLUT-4 glucose transporter in adipocytes from hypertensive rats. Metabolism 2004; 53:382-7.

37. Katayama S, Inaba M, Maruno Y, Morita T, Awata T, Oka Y. Glucose intolerance in spontaneously hypertensive and Wistar-Kyoto rats: Enhanced gene expression and synthesis of skeletal muscle glucose transporter 4 . Hypertens Res 1997;20:279-86.

38. Okamoto MM, Sumida DH, Carvalho CR, Vargas $A M$, Heimann JC, Schaan BD, et al. Changes in dietary sodium consumption modulate GLUT4 gene expression and early steps of insulin signaling. Am J Physiol Regul Integr Comp Physiol 2004;286:R779-85.

39. Goodyear LJ, Kahn BB. Exercise, glucose transport, and insulin sensitivity. Annu Rev Med 1998;49:235-61.

40. Kramer D, Shapiro R, Adler A, Bush E, Rondinone CM. Insulin-sensitizing effect of rosiglitazone (BRL-49653) by regulation of glucose transporters in muscle and fat of Zucker rats. Metabolism 2001;50:1294-300.

41. Al-Khalili L, Forsgren M, Kannisto K, Zierath JR, Lonnqvist F, Krook A. Enhanced insulin-stimulated glycogen synthesis in response to insulin, metformin or rosiglitazone is associated with increased mRNA expression of GLUT4 and peroxisomal proliferator activator receptor gamma coactivator 1. Diabetologia 2005;48:1173-9.

42. da Costa Lima NK, Lima FB, dos Santos EA, Okamoto MM, Matsushita DH, Hell NS, et al. Chronic salt overload increases blood pressure and improves glucose metabolism without changing insulin sensitivity. Am J Hypertens 1997; 10(7P+1):720-7.

43. Prada P, Okamoto MM, Furukawa LN, Machado UF, Heimann JC, Dolnikoff MS. High- or low-salt diet from weaning to adulthood: Effect on insulin sensitivity in Wistar rats. Hypertension 2000;35(1P+2):424-9.
44. Liu ML, Gibbs EM, McCoid SC, Milici AJ, Stukenbrok HA, McPherson RK, et al. Transgenic mice expressing the human GLUT4/muscle-fat facilitative glucose transporter protein exhibit efficient glycemic control. Proc Natl Acad Sci USA 1993;90:11346-50.

45. Silva JL, Giannocco G, Furuya DT, Lima GA, Moraes PA, Nachef $S$, et al. NF-kappaB, MEF2A, MEF2D and HIF1-a involvement on insulin- and contraction-induced regulation of GLUT4 gene expression in soleus muscle. Mol Cell Endocrinol 2005;240(1-2):82-93.

46. Thorens B. Facilitated glucose transporters in epithelial cells. Annu Rev Physiol 1993:55:591-608.

47. Im SS, Kang SY, Kim SY, Kim HI, Kim JW, Kim KS, et al. Glucose-stimulated upregulation of GLUT2 gene is mediated by sterol response element-binding protein-1c in the hepatocytes. Diabetes 2005;54:1684-91.

48. Powell EE, Jonsson JR, Clouston AD. Dangerous liaisons: the metabolic syndrome and nonalcoholic fatty liver disease. Ann Intern Med 2005; 143:753-4.

49. Hamaguchi M, Kojima T, Takeda N, Nakagawa T, Taniguchi $\mathrm{H}$, Fujii $\mathrm{K}$, et al. The metabolic syndrome as a predictor of nonalcoholic fatty liver disease. Ann Intern Med 2005; 143:722-8.

50. Samuel VT, Liu ZX, Qu X, Elder BD, Bilz S, Befroy D, et al. Mechanism of hepatic insulin resistance in non-alcoholic fatty liver disease. J Biol Chem 2004;279:32345-53.

51. Okamoto Y, Tanaka S, Haga Y. Enhanced GLUT2 gene expression in an oleic acid-induced in vitro fatty liver model. Hepatol Res 2002;23:138-44.

52. Tomie Furuya D, Binsack R, Onishi ME, Monteiro Seraphim P, Fabres Machado U. Low ethanol consumption induces enhancement of insulin sensitivity in liver of normal rats. Life Sci 2005;77:1813-24.

53. DeFronzo RA, Ferrannini E. Insulin resistance. A multifaceted syndrome responsible for NIDDM, obesity, hypertension, dyslipidemia, and atherosclerotic cardiovascular disease. Diabetes Care 1991;14:173-94.

54. Reddy S, Young M, Poole CA, Ross JM. Loss of glucose transporter-2 precedes insulin loss in the nonobese diabetic and the low-dose streptozotocin mouse models: A comparative immunohistochemical study by light and confocal microscopy. Gen Comp Endocrinol 1998; 111:9-19.

55. Picinato MC, Curi R, Machado UF, Carpinelli AR. Soybean- and olive-oils-enriched diets increase insulin secretion to glucose stimulus in isolated pancreatic rat islets. Physiol Behav 1998;65:289-94.

56. Kim HI, Kim JW, Kim SH, Cha JY, Kim KS, Ahn YH. Identification and functional characterization of the peroxisomal proliferator response element in rat GLUT2 promoter. Diabetes 2000;49:1517-24.

57. Brosius 3rd FC, Nguyen N, Egert S, Lin Z, Deeb GM, Haas $\mathrm{F}$, et al. Increased sarcolemmal glucose transporter abundance in myocardial ischemia. Am J Cardiol 1997:80(3A):77A-84A.

58. Simões MV, Egert S, Ziegler S, Miyagawa M, Reder S, Lehner $\mathrm{T}$, et al. Delayed response of insulin-stimulated fluorine-18 deoxyglucose uptake in glucose transporter4-null mice hearts. J Am Coll Cardiol 2004;43:1690-7. 
59. Brosius 3rd FC, Liu Y, Nguyen N, Sun D, Bartlett J, Schwaiger M. Persistent myocardial ischemia increases GLUT1 glucose transporter expression in both ischemic and non-ischemic heart regions. J Mol Cell Cardiol 1997:29:1675-85

60. Kraegen EW, Sowden JA, Halstead MB, Clark PW, Rodnick KJ, Chisholm DJ, et al. Glucose transporters and in vivo glucose uptake in skeletal and cardiac muscle: Fasting, insulin stimulation and immunoisolation studies of GLUT1 and GLUT4. Biochem J 1993;295 (P+1):287-93.

61. Luiken JJ, Coort SL, Koonen DP, Bonen A, Glatz JF. Signalling components involved in contraction-inducible substrate uptake into cardiac myocytes. Proc Nutr Soc 2004:63:251-8.

62. Russell 3rd RR, Bergeron R, Shulman GI, Young LH. Translocation of myocardial GLUT-4 and increased glucose uptake through activation of AMPK by AICAR. Am J Physiol 1999:277(2P+2):H643-9.

63. Egert S, Nguyen N, Brosius 3rd FC, Schwaiger M. Effects of wortmannin on insulin- and ischemia-induced stimulation of GLUT4 translocation and FDG uptake in perfused rat hearts. Cardiovasc Res 1997:35:283-93.

64. Belke DD, Larsen TS, Gibbs EM, Severson DL. Altered metabolism causes cardiac dysfunction in perfused hearts from diabetic ( $\mathrm{db} / \mathrm{db})$ mice. Am J Physiol Endocrinol Metab 2000:279:E1 104-13.

65. Vettor $R$, Fabris $R$, Serra $R$, Lombardi AM, Tonello $C$, Granzotto M, et al. Changes in FAT/CD36, UCP2, UCP3 and GLUT4 gene expression during lipid infusion in rat skeletal and heart muscle. Int J Obes Relat Metab Disord 2002:26:838-47.

66. Morel S, Berthonneche C, Tanguy S, Toufektsian MC, Perret $\mathrm{P}$, Ghezzi C, et al. Early pre-diabetic state alters adaptation of myocardial glucose metabolism during ischemia in rats. Mol Cell Biochem 2005;272(1-2):9-17.

67. Chinali M, Devereux RB, Howard BV, Roman MJ, Bella $\mathrm{JN}$, Liu JE, et al. Comparison of cardiac structure and function in American Indians with and without the metabolic syndrome (the Strong Heart Study). Am J Cardiol 2004:93:40-4

68. Rutter MK, Parise $H$, Benjamin EJ, Levy D, Larson MG, Meigs JB, et al. Impact of glucose intolerance and insulin resistance on cardiac structure and function: Sexrelated differences in the Framingham Heart Study. Circulation 2003; 107:448-54.

69. Katz EB, Stenbit AE, Hatton K, DePinho R, Charron MJ. Cardiac and adipose tissue abnormalities but not diabetes in mice deficient in GLUT4. Nature 1995;377:151-5.

70. Sidell RJ, Cole MA, Draper NJ, Desrois M, Buckingham RE, Clarke K. Thiazolidinedione treatment normalizes insulin resistance and ischemic injury in the zucker Fatty rat heart. Diabetes 2002;51:1110-7.

71. Bahr M, Spelleken M, Bock M, von Holtey M, Kiehn R, Eckel J. Acute and chronic effects of troglitazone (CS045) on isolated rat ventricular cardiomyocytes. Diabetologia 1996;39:766-74.

72. Park JL, Loberg RD, Duquaine D, Zhang H, Deo BK, Ardanaz N, et al. GLUT4 facilitative glucose transporter specifically and differentially contributes to agonistinduced vascular reactivity in mouse aorta. Arterioscler Thromb Vasc Biol 2005:25:1596-602.
73. Atkins KB, Johns D, Watts S, Clinton Webb R, Brosius 3rd FC. Decreased vascular glucose transporter expression and glucose uptake in DOCA-salt hypertension. J Hypertens 2001;19:1581-7.

74. Atkins KB, Northcott CA, Watts SW, Brosius FC. Effects of PPAR-gamma ligands on vascular smooth muscle marker expression in hypertensive and normal arteries. Am J Physiol Heart Circ Physiol 2005;288:H235-43.

75. Bergandi L, Silvagno F, Russo I, Riganti C, Anfossi G, Aldieri $\mathrm{E}$, et al. Insulin stimulates glucose transport via nitric oxide/cyclic GMP pathway in human vascular smooth muscle cells. Arterioscler Thromb Vasc Biol 2003:23:2215-21.

76. Karam JH. Hypoglycemic disorders. In: Greenspan FS, Gardner DG, eds. Basic \& clinical endocrinology. $5^{\text {th }}$ edition. New York:Mc Graw-Hill; 2001. p.701.

77. Fernandes R, Suzuki K, Kumagai AK. Inner blood-retinal barrier GLUT1 in long-term diabetic rats: an immunogold electron microscopic study. Invest Ophthalmol Vis Sci 2003:44:3150-4.

78. Antonetti DA, Barber AJ, Khin S, Lieth E, Tarbell JM, Gardner TW. Vascular permeability in experimental diabetes is associated with reduced endothelial occludin content: Vascular endothelial growth factor decreases occludin in retinal endothelial cells. Penn State Retina Research Group. Diabetes 1998;47:1953-9.

79. Gaudreault N, Scriven DR, Moore ED. Characterization of glucose transporters in the intact coronary artery endothelium in rats: GLUT-2 upregulated by long-term hyperglycaemia. Diabetologia 2004;47:2081-92.

80. Wright EM. Renal Na(+)-glucose cotransporters. Am J Physiol Renal Physiol 2001;280:F10-8.

81. Vestri S, Okamoto MM, de Freitas HS, Aparecida dos Santos R, Nunes MT, Morimatsu M, et al. Changes in sodium or glucose filtration rate modulate expression of glucose transporters in renal proximal tubular cells of rat. $\mathbf{J}$ Membr Biol 2001;182:105-12.

82. Schaan BD, Irigoyen MC, Lacchini S, Moreira ED, Schmid $H$, Machado UF. Sympathetic modulation of the renal glucose transporter GLUT2 in diabetic rats. Auton Neurosci 2005; 117:54-61.

83. Chin $\mathrm{E}$, Zamah $\mathrm{AM}$, Landau $\mathrm{D}$, Gronbcek $\mathrm{H}$, Flyvbjerg $\mathrm{A}$, LeRoith $D$, et al. Changes in facilitative glucose transporter messenger ribonucleic acid levels in the diabetic rat kidney. Endocrinology 1997;138:1267-75

84. Dominguez JH, Camp K, Maianu L, Garvey WT. Glucose transporters of rat proximal tubule: Differential expression and subcellular distribution. Am J Physiol 1992;262(5P+2):F807-12

85. Freitas HS, Schaan BD, Seraphim PM, Nunes MT, Machado UF. Acute and short-term insulin-induced molecular adaptations of GLUT2 gene expression in the renal cortex of diabetic rats. Mol Cell Endocrinol 2005:237(1-2):49-57.

86. D'Agord Schaan B, Lacchini S, Bertoluci MC, Irigoyen MC, Machado UF, Schmid H. Impact of renal denervation on renal content of GLUT1, albuminuria and urinary TGF-betal in streptozotocin-induced diabetic rats. Auton Neurosci 2003:104:88-94. 
87. Zhang J, Liu Z, Liu D, Li L. Identification of glucose transporter- 1 and its functional assay in mouse glomerular mesangial cells cultured in vitro. Chin Med Sci J 2001;16:35-9.

88. Li Y, Liu Z, Liu D, Zhang J, Chen Z, Li L. Identification and function of glucose transporter 1 in human mesangial cells. Chin Med J (Engl) 2001;1 14:824-8.

89. D'Agord Schaan B, Lacchini S, Bertoluci MC, Irigoyen MC, Machado UF, Schmid H. Increased renal GLUTI abundance and urinary TGF-beta 1 in streptozotocininduced diabetic rats: Implications for the development of nephropathy complicating diabetes. Horm Metab Res 2001;33:664-9.

90. Schaan BD, Irigoyen MC, Bertoluci MC, Lima NG, Passaglia J, Hermes E, et al. Increased urinary TGF-betal

and cortical renal GLUT1 and GLUT2 levels: Additive effects of hypertension and diabetes. Nephron Physiol 2005; 100:43-50.

91. Huggett RJ, Burns J, Mackintosh AF, Mary DA. Sympathetic neural activation in nondiabetic metabolic syndrome and its further augmentation by hypertension. Hypertension 2004;44:847-52.

\section{Endereço para correspondência:}

Ubiratan Fabres Machado

Departamento de Fisiologia e Biofísica, ICB/USP

Av. Prof. Lineu Prestes 1524

05508-900 São Paulo, SP

Fax: (1 1) 3091-7494

E-mail: ubiratan@icb.usp.br 